\title{
The risk of reoperative cardiac surgery in radiation-induced valvular disease
}

\author{
Julius I. Ejiofor, MD, MPH, ${ }^{\mathrm{a}}$ Fernando Ramirez-Del Val, MD, MPH, ${ }^{\mathrm{a}}$ Anju Nohria, MD, ${ }^{\mathrm{b}}$ \\ Anthony Norman, BS, ${ }^{a}$ Siobhan McGurk, BS, ${ }^{a}$ Sary F. Aranki, MD, ${ }^{a}$ Prem Shekar, MD, ${ }^{a}$ \\ Lawrence H. Cohn, MD, ${ }^{a}$ and Tsuyoshi Kaneko, MD $^{\mathrm{a}}$
}

\section{ABSTRACT}

Objective: Mediastinal radiation therapy (MRT) increases the risk for adverse outcomes after cardiac surgery and is not incorporated in the Society of Thoracic Surgeons (STS) risk algorithm. We aimed to quantify the surgical risk conferred by MRT in patients undergoing primary and reoperative valvular operations.

Methods: A retrospective analysis of 261 consecutive patients with prior MRT who underwent valvular operations between January 2002 and May 2015. Short- and long-term outcomes were compared for STS predicted risk of mortality, surgery type, gender, year of surgery, and age-matched patients stratified by reoperative status.

Results: Mean age was $62.6 \pm 12.1$ years and $174(67 \%)$ were women. The majority had received MRT for Hodgkin lymphoma (48.2\%) and breast cancer $(36 \%)$. Overall, $214(82 \%)$ were primary and $47(18 \%)$ were reoperative procedures. Reoperation carried a higher operative mortality than primary cases $(17 \%$ vs $3.7 \% ; P=.003)$. Compared with the 836 nonradiated matches, operative mortality and observed-to-expected STS mortality ratios were higher in primary (3.8\% [1.4] vs $0.8 \%$ [0.32]; $P=.004)$ and reoperative $(17 \%$ [3.35] vs $2.3 \%$ $[0.45] ; P=.001)$ patients with prior MRT. Cox proportional hazard modeling revealed that in patients with previous MRT, primary (hazard ratio, 2.24; 95\% confidence interval, 1.73-2.91) and reoperative status (hazard ratio, 3.19; 95\% confidence interval, 1.95-5.21) adversely affected long-term survival compared with nonradiated matches.

Conclusions: Surgery for radiation-induced valvular heart disease has a higher operative mortality than predicted by STS predicted risk of mortality. Reoperations are associated with increased morbidity and mortality compared with primary cases. Careful patient selection is paramount and expanded indications for transcatheter therapies should be considered, especially in reoperative patients. (J Thorac Cardiovasc Surg 2017;154:1883-95)

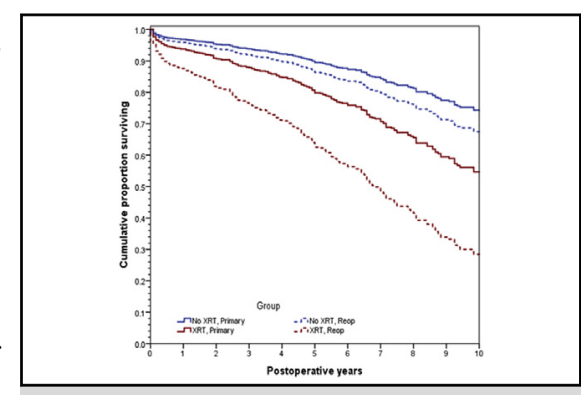

Reoperative status was a significant predictor for long term mortality (hazard ratio, 1.92; $P=.013$ ).

\section{Central Message}

Surgery for radiation-induced valvular hear disease has higher mortality than predicted by Society of Thoracic Surgeons score. Reoperations are associated with increased morbidity and mortality compared with primary cases.

\section{Perspective}

This analysis of patients undergoing surgery for radiation-induced valvular heart disease shows that the Society of Thoracic Surgeons risk model does not adequately capture their operative mortality. Reoperation for radiationinduced valvular heart disease is associated with a higher operative and long-term mortality compared with primary surgery. This result should prompt careful preoperative risk evaluation and expand the indications for transcatheter valve therapies.

See Editorial Commentary page 1896.

\footnotetext{
From the Divisions of aCardiac Surgery and 'Cardiology, Brigham and Women's Hospital, Harvard Medical School, Boston, Mass.

Supported by departmental funds.

J.I.E. and F.R-D.V. contributed equally to this article.

Read at the 96th Annual Meeting of The American Association for Thoracic Surgery, Baltimore, Maryland, May 14-18, 2016.

Received for publication Sept 27, 2016; revisions received June 14, 2017; accepted for publication July 3, 2017; available ahead of print Sept 1, 2017.

Address for reprints: Tsuyoshi Kaneko, MD, Division of Cardiac Surgery, Brigham and Women's Hospital, 75 Francis St, Boston, MA 02115 (E-mail: tkaneko2@ partners.org).

$0022-5223 / \$ 36.00$

Copyright (C) 2017 by The American Association for Thoracic Surgery

http://dx.doi.org/10.1016/j.jtcvs.2017.07.033
}

Mediastinal radiation therapy (MRT) remains an effective treatment for thoracic and chest wall neoplasms, including lymphomas, breast cancer, thymomas, and lung and esophageal cancers. However, MRT carries many delayed cardiovascular adverse effects such as accelerated coronary

Scanning this QR code will take you to the article title page.

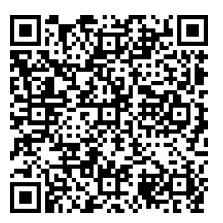




\section{Abbreviations and Acronyms \\ AVR = aortic valve replacement \\ EMR = electronic medical record \\ MRT = mediastinal radiation therapy \\ NYHA $=$ New York Heart Association \\ PROM $=$ predicted risk of mortality \\ STS $=$ Society of Thoracic Surgeons \\ VHD $=$ valvular heart disease}

artery disease, valvular heart disease (VHD), conduction system abnormalities, pericardial constriction, and restrictive cardiomyopathy. ${ }^{1-6}$ This risk is increased with extended radiation treatment fields and higher radiation doses, such as those used to treat Hodgkin lymphoma, testicular cancer, and thymomas. ${ }^{7}$ VHD is estimated to develop in $6 \%$ to $15 \%{ }^{8}$ of patients who receive MRT, 11 to 16 years after its completion. ${ }^{2,9}$ Aortic regurgitation, aortic stenosis, and tricuspid regurgitation are present in $60 \%, 16 \%$, and $4 \%$ of these patients, respectively. ${ }^{10,11}$ Aortic stenosis is the most common MRT-induced valvular abnormality requiring surgical intervention. ${ }^{10}$

It is known that prior MRT is associated with inferior short-term and long-term outcomes after cardiac surgery. $5,7,12,13$ However, the most commonly used risk prediction algorithm in the United States, the Society of Thoracic Surgeons (STS) predicted risk of mortality (PROM), does not incorporate prior MRT into its calculations. ${ }^{14,15}$ Although there are several reports evaluating operative outcomes for radiation-induced VHD, they are limited by their small numbers and heterogeneous cohorts. ${ }^{3,5,13,16}$ We hypothesized that surgery for radiation-induced VHD is associated with adverse outcomes and that this risk is amplified in patients undergoing reoperative cardiac surgery. Accordingly, we compared the outcomes of patients undergoing primary and reoperative valvular operations for radiation-induced VHD to a matched cohort of primary and reoperative valvular patients without a history of MRT.

\section{METHODS \\ Study Population}

All adult patients aged $\geq 18$ years who underwent valvular surgery at Brigham and Women's Hospital between January 2002 and May 2015 were identified from our electronic medical record (EMR). All patients with isolated valvular or concomitant valvular and/or coronary artery bypass graft surgery were included. Those with valvular operations for endocarditis, heart transplantation, or ventricular assist devices were excluded. A total of 261 out of 9659 (2.7\%) patients underwent valvular surgery for VHD following MRT for a documented malignancy. Patients were grouped by whether it was a primary cardiac surgery $(n=214)$ or a redosternotomy (reoperation) $(\mathrm{n}=47)$.

The indication for MRT was collected for all patients. However, the dates and doses of MRT were not reliably documented in the available EMR, predominately due to the remoteness of the MRT. The diagnosis of MRT-induced VHD was based on comprehensive clinical and imaging data as well as the expert opinion of the referring cardiologist. All patients were cleared by oncology before surgery.

\section{Data Collection and Outcomes}

Patient characteristics, perioperative data, laboratory test results, and in-hospital outcomes were recorded electronically at the time of presentation. Data were extracted from the EMR and variables were defined and coded according to the STS Adult Cardiac Surgery Database version 2.52 specifications, unless otherwise noted. The presence of meaningful coronary artery disease was defined as a $\geq 70 \%$ obstruction in any coronary or $\geq 50 \%$ in the left main coronary artery. Right ventricular dysfunction, right ventricular dilation, nonischemic cardiomyopathy, and restrictive cardiomyopathy were deemed positive if present as moderate or severe in the patients' preoperative echocardiogram. Operative mortality was defined as any death occurring in-house during the index admission, or within 30 days of surgery if discharged.

The primary outcomes of interest were operative mortality and long-term survival. Secondary outcomes included valve-related complications, perioperative morbidity, and length of stay.

Longitudinal mortality data was obtained from our internal research repository, routine patient follow-up, and our state Department of Public Health. Survival follow-up was $100 \%$ in this cohort. Follow-up time was measured in months from the date of surgery to the date of death or study end (April 30, 2016) if alive.

Partners Healthcare Institutional Review Board approved this study. Informed consent from individual patients was waived.

\section{Statistical Analysis}

Normally distributed continuous variables are expressed as mean \pm standard deviation and were compared using Student $t$ tests with Levene test for homogeneity of variance. Nonnormally distributed variables are expressed as median and interquartile range and were compared using Mann-Whitney $U$ tests. Categorical variables are presented as number and percentage and were compared using $\chi^{2}$ or Fisher exact tests. Longitudinal survival was estimated by Kaplan-Meier analyses, and groups were compared using log-rank test. A forward-stepwise Cox proportional hazard model was used to evaluate risk factors influencing survival. Continuous variables evaluated included age, creatinine (in milligrams per deciliter), and ejection fraction (as a percentage). Categorical variables included type of cancer, type of surgery, gender, vascular and cerebrovascular disease, renal insufficiency, congestive heart failure, and previous cardiac surgery. Variables selected for inclusion in the Cox model included those that were significantly associated with mortality on univariate analyses, those known to be contributors to all-cause mortality, and those with clinical importance to morbidity and mortality. All analyses were conducted using IBM SPSS Statistics version 22.0 (IBM-SPSS Inc, Armonk, NY) and $P \leq .05$ was the criterion for significance.

\section{Matched Analysis}

To better quantify the operative morbidity and mortality risks associated with MRT before reoperative valvular surgery, we sought to compare outcomes with a matched cohort. We thus first derived from our consecutive series of valve patients a pool of potential matches with the same frequency distributions for age, gender, year of surgery, and type of procedure, who had no documented history of cancer. We then selected matches on the basis of nearest STS PROM within a $0.05 \%$ caliper, followed by surgery type, gender, year of surgery, and age, stratified by reoperative status. The intended goal was 3 to 4 matches per MRT patient; however, all matches with exact STS PROM (to 4 decimal places), procedure, and gender were retained. A total of 1049 matched patients were identified; 836 primary patients without MRT (3.2 matches per MRT patient) and 213 reoperative patients (5.7 matches per reoperative 
TABLE 1. Baseline characteristics of primary and reoperative patients with radiation-induced valvular heart disease

\begin{tabular}{|c|c|c|c|c|}
\hline Demographic characteristic & All patients $(n=261)$ & Reoperative $(n=47)$ & Primary $(n=214)$ & $P$ value \\
\hline Age (y) & $62.6 \pm 12.1$ & $60.5 \pm 13.5$ & $63.1 \pm 12.1$ & .178 \\
\hline Women & $174(66.6)$ & $19(40.4)$ & $155(72.4)$ & .001 \\
\hline Diabetes & $47(18.0)$ & $13(27.7)$ & $34(15.9)$ & .062 \\
\hline Renal insufficiency & $15(5.7)$ & $6(12.8)$ & $9(4.2)$ & .034 \\
\hline Preoperative Cr (mg/dL) & $1.15 \pm 0.38$ & $1.23 \pm 0.57$ & $0.98 \pm 0.43$ & .001 \\
\hline Peripheral vascular disease & $33(12.6)$ & $13(27.7)$ & $20(9.3)$ & .002 \\
\hline Cerebrovascular accident & $5(1.9)$ & $3(6.4)$ & $2(0.9)$ & .042 \\
\hline Endocarditis & $9(3.5)$ & $5(10.6)$ & $4(1.9)$ & .011 \\
\hline Congestive heart failure & $133(51.0)$ & $32(68.3)$ & $101(47.2)$ & .053 \\
\hline NYHA class III or IV & $104(39.8)$ & $27(57.4)$ & $77(36.0)$ & .008 \\
\hline Ejection fraction $(\%)$ & $60.0(50-65)$ & $55.0(45-60)$ & $60.0(50-65)$ & .009 \\
\hline Atrial fibrillation & $24(9.2)$ & $19(8.9)$ & $5(10.6)$ & .780 \\
\hline STS PROM & $3.01 \pm 3.05$ & $5.21 \pm 5.37$ & $2.58 \pm 2.15$ & .001 \\
\hline \multicolumn{5}{|l|}{ Preoperative cardiac function } \\
\hline Coronary artery disease & $111(42.5)$ & 85 (39.7) & $26(55.3)$ & .072 \\
\hline Left coronary artery & $86(32.9)$ & $64(29.9)$ & $22(46.8)$ & .039 \\
\hline Right coronary artery & $69(26.5)$ & $47(22)$ & $22(46.8)$ & .002 \\
\hline Left main disease & $31(11.8)$ & $23(10.7)$ & $8(17)$ & .222 \\
\hline Nonischemic cardiomyopathy & $20(7.7)$ & $16(7.5)$ & $4(8.5)$ & .765 \\
\hline Restrictive cardiomyopathy & $4(1.5)$ & $3(1.4)$ & $1(2.1)$ & .550 \\
\hline Moderate/severe RV dysfunction & $6(2.3)$ & $2(0.9)$ & $4(8.6)$ & .011 \\
\hline Moderate/severe RV dilation & $11(4.2)$ & $6(2.8)$ & $5(10.6)$ & .030 \\
\hline Moderate/severe tricuspid regurgitation & $50(19.1)$ & $36(16.8)$ & $14(29.8)$ & .063 \\
\hline
\end{tabular}

Values are presented as $\mathrm{n}(\%)$, mean \pm standard deviation, or median (IQR). Cr, Creatinine; NYHA, New York Heart Association; STS, Society of Thoracic Surgeons; $P R O M$, predicted risk of mortality; $R V$, right ventricle

MRT patient). Three MRT patients could not be matched satisfactorily. Conditional models using logistic regression for operative mortality and a Cox proportional hazards for cumulative survival were performed to adjust for remaining differences in the matched cohorts baseline characteristics.

\section{RESULTS}

\section{Preoperative Characteristics of Patients With Radiation-Induced VHD}

Baseline characteristics and preoperative cardiac function of the overall cohort of primary and reoperative patients with radiation-induced VHD are shown in Table 1. Although age was similar between primary and reoperative patients (63.1 vs 60.5 years, respectively; $P=.18)$, there were more women in the primary surgical group $(72.4 \%$ vs $40.4 \% ; P=.001)$. Reoperative patients had more comorbidities, including renal insufficiency $(12.8 \%$ vs $4.2 \% ; P=.034)$, peripheral vascular disease $(27.7 \%$ vs $9.3 \% ; P=.002)$, prior stroke $(6.4 \%$ vs $0.9 \%$; $P=.042)$, and previous history of endocarditis $(10.6 \%$ vs $1.9 \% ; P=.011)$. The overall prevalence of atrial fibrillation was $9.2 \%(10 \%$ in reoperative and $8.9 \%$ in primary cases; $P=.78$ ) The mean STS PROM was significantly higher for reoperative patients with those undergoing primary procedures (5.21 vs $2.58 ; P=.001)$.

The overall prevalence of coronary artery disease was $42 \%-32.9 \%$ on the left and $26.5 \%$ on the right coronary artery-with $11.8 \%$ having $>50 \%$ obstruction in the left main coronary artery. Patients undergoing reoperation had a higher burden of both left $(46.8 \%$ vs $29.9 \% ; P=.039)$ and right $(46.8 \%$ vs $22 \% ; P=.002)$ coronary disease, compared with primary cases.

Pericarditis was present in $10.7 \%$ of patients, $2.7 \%$ of which was constrictive. Moderate or severe right ventricular dysfunction was observed in $2.3 \%$ of the cohort, but was higher among reoperative cases $(8.6 \%$ vs $0.9 \%$; $P=.011$ ). Moderate or severe right ventricular dilation was present in $4.2 \%$ of patients and was also higher among reoperative cases $(10.6 \%$ vs $2.8 \% ; P=.030)$.

The malignancies for which MRT was indicated are shown in Table 2. Hodgkin lymphoma was the most common overall $(48.3 \%)$, but was more common in the reoperative group $(61.7 \%$ vs $45.3 \% ; P=.053)$. Breast cancer was the second most common malignancy $(36 \%)$, 
TABLE 2. Previous malignancy for reoperative and primary patients with radiation-induced valvular heart disease

\begin{tabular}{|c|c|c|c|c|}
\hline Previous malignancy & All patients $(n=261)$ & Reoperative $(n=47)$ & Primary $(n=214)$ & $P$ value \\
\hline Breast cancer & $94(36.0)$ & $8(17.0)$ & $86(40.2)$ & .002 \\
\hline Hodgkin lymphoma & $126(48.3)$ & $29(61.7)$ & $97(45.3)$ & .053 \\
\hline Testicular/thymic & $6(2.3)$ & $1(2.1)$ & $5(2.3)$ & 1.000 \\
\hline Other & $35(13.4)$ & $9(19.1)$ & $26(12.1)$ & .236 \\
\hline Adenoid carcinoma & $1(0.4)$ & $0(0.0)$ & $1(0.5)$ & \\
\hline Esophageal & $4(1.5)$ & $2(4.3)$ & $2(0.9)$ & \\
\hline Germ cell tumor & $1(0.4)$ & $0(0.0)$ & $1(0.5)$ & \\
\hline Head and neck & $1(0.4)$ & $0(0.0)$ & $1(0.5)$ & \\
\hline Laryngeal & $1(0.4)$ & $0(0.0)$ & $1(0.5)$ & \\
\hline Leukemia & $1(0.4)$ & $1(2.1)$ & $0(0.0)$ & \\
\hline Lung/bronchus & $6(2.3)$ & $1(2.1)$ & $5(2.3)$ & \\
\hline Neuroblastoma & $1(0.4)$ & $1(2.1)$ & $0(0.0)$ & \\
\hline Non-Hodgkin lymphoma & $11(4.3)$ & $1(2.1)$ & $10(4.8)$ & \\
\hline Ovarian & $1(0.4)$ & $0(0.0)$ & $1(0.5)$ & \\
\hline Prostate, metastatic & $1(0.4)$ & $1(2.1)$ & $0(0.0)$ & \\
\hline Sarcoma & $2(0.7)$ & $0(0.0)$ & $2(0.9)$ & \\
\hline Seminoma & $2(0.7)$ & $0(0.0)$ & $2(0.9)$ & \\
\hline Throat & $2(0.8)$ & $2(4.3)$ & $0(0.0)$ & \\
\hline
\end{tabular}

Values are presented as $\mathrm{n}(\%)$.

but was significantly more prevalent in the primary group (40.2\% vs $17 \% ; P=.002)$. The remaining $15.7 \%$ of patients had a variety of malignancies. None of the patients had a documented recurrence at the time of valvular surgery.

\section{Operative Variables of Patients With Radiation- Induced VHD}

The indications for surgery and the types of procedures performed are shown in Table 3. Altogether, 77\% (202 out of 261) of patients underwent an aortic valve replacement (AVR) $(79 \%$ of primary vs $68 \%$ of reoperations; $P=.12$ ). The most common procedure was isolated AVR, constituting $31 \%$ of the primary and $27 \%$ of the reoperative group, respectively. Most concomitant surgeries were a combination of AVR with coronary artery bypass graft in $20.3 \%$ or AVR with a second valve in $8 \%$ of all patients. Complex valvular procedures comprised $18.3 \%$ of our series and isolated mitral valve surgery was performed in $15 \%$ of patients in both groups. There was no difference in the distribution frequencies of all surgical procedures between primary and reoperative cases $(P>.55)$.

Reoperations had longer median cardiopulmonary bypass time (248 minutes vs 131 minutes) and crossclamp time (145 minutes vs 92 minutes) compared with the primary group (both $P$ values $\leq .001$ ). More patients were transfused with packed red blood cells $(59 \%$ vs $32 \%$; $P=.001)$, fresh frozen plasma $(53 \%$ vs $8.4 \%$; $P=.001)$, and platelets $(48 \%$ vs $7.9 \% ; P=.001)$ among patients undergoing reoperation (Table 3 ).

\section{Postoperative Outcomes of Patients With Radiation- Induced VHD}

Reoperative group was at greater risk of returning to the operating room $(8.5 \%$ vs $1.9 \% ; P=.037)$ (Table 4$)$. They also had a higher incidence of new onset renal dysfunction and postoperative transfusions. The median time on a ventilator (20.3 hours vs 7 hours), length of stay in the intensive care unit (122 hours vs 51 hours), and overall hospital length of stay ( 15 days vs 7 days) were significantly longer in the reoperative group (all $P$ values $=.001$ ).

Operative mortality for the entire cohort was $6.1 \%$ (16 out of 261) and was significantly higher in reoperative cases $(17 \%$ vs $3.7 \% ; P=.003)$ (Table 4$)$. The observed to expected STS mortality ratio (observed-to-expected STS) was 3.2 (17 out of 5.2) for reoperative and 1.4 (3.7 out of 2.6) for primary patients. There was no difference in operative mortality based on the underlying history of cancer: $8.5 \%$ ( 8 out of 94 ) for breast cancer versus $6.1 \%$ (8 out of 132) for Hodgkin/thymic/testicular cancer $(P=.60)$.

\section{Long-term Survival of Patients With Radiation- Induced VHD}

Median follow-up was 3.8 years (IQR, 1.8-6.7; range, 0-13.8 years), for a total of 1149 patient-years, and there were 79 deaths during follow-up observation. Estimated overall survival was $55 \%$ at 10 years, and observed survival was significantly higher for primary patients (mean, 9.5 years; $95 \%$ confidence interval $[\mathrm{CI}], 8.7-10.4)$ than for reoperative cases (5.7 years; $95 \%$ CI, 4.1-7.6; 
TABLE 3. Operative data for reoperative and primary patients with radiation-induced valvular heart disease

\begin{tabular}{|c|c|c|c|c|}
\hline Operative data & All patients $(n=261)$ & Reoperative $(n=47)$ & Primary $(n=214)$ & $P$ value \\
\hline Valve procedures & & & & .552 \\
\hline Isolated aortic valve & $80(30.7)$ & $13(27.7)$ & $67(31.3)$ & \\
\hline Aortic valve + coronary artery bypass graft & $53(20.3)$ & $6(12.8)$ & $47(22.0)$ & \\
\hline Aortic valve + other valve & $21(8.0)$ & $3(6.4)$ & $18(8.4)$ & \\
\hline Isolated mitral valve & $39(15.0)$ & $7(14.9)$ & $32(15.0)$ & \\
\hline Other isolated valve & $5(1.9)$ & $4(8.1)$ & $1(0.5)$ & \\
\hline Other valve + coronary artery bypass graft & $15(5.7)$ & $4(8.5)$ & $11(5.1)$ & \\
\hline Miscellaneous complex valve & $48(18.3)$ & $10(21.3)$ & $38(17.7)$ & \\
\hline \multicolumn{5}{|l|}{ Indication for surgery } \\
\hline Aortic insufficiency & $40(15.3)$ & $9(19.1)$ & $31(14.5)$ & \\
\hline Aortic stenosis & $41(15.7)$ & $7(14.9)$ & $34(15.9)$ & \\
\hline Aortic insufficiency/aortic stenosis & $136(52.1)$ & $16(34.1)$ & $120(56.1)$ & \\
\hline Other valve indication & $44(16.9)$ & $15(31.9)$ & $29(13.6)$ & \\
\hline Perfusion time (min) & $142(102-215)$ & $248(191-390)$ & $131(98-180)$ & .001 \\
\hline Crossclamp time (min) & $98(74-141)$ & $145(94-212)$ & $92(67-133)$ & .001 \\
\hline \multicolumn{5}{|l|}{ Patients transfused in operating room } \\
\hline Pack of red blood cells & $98(37.5)$ & $28(59.6)$ & $70(32.7)$ & .001 \\
\hline Fresh frozen plasma & $43(16.5)$ & $25(53.2)$ & $18(8.4)$ & .001 \\
\hline Platelets & $40(15.3)$ & $23(48.8)$ & $17(7.9)$ & .001 \\
\hline
\end{tabular}

Values are presented as $\mathrm{n}(\%)$ or median (interquartile range).

$P<.001$ ) (Figure 1). We did not observe a significant difference in postoperative survival between patients with a history of breast cancer (mean, 9.1 years; 95\% CI, 7.851-10.378) and those with a history of Hodgkin/ thymic/testicular cancer (mean, 8.9 years; 95\% CI, 7.9-10.1; $P=.829$ ).

\section{Matched Analysis of Primary and Reoperative Patients With and Without Prior MRT}

The matched cohorts of primary (836 without MRT and 213 with MRT) and reoperative (213 without MRT and 46 with MRT) valve surgery patients were well balanced. Both primary and reoperative matched cohorts had similar baseline characteristics, including STS PROM, left ventricular ejection fraction, New York Heart Association (NYHA) class III or IV, renal insufficiency, and diabetes (Table 5). However, primary matched patients with MRT were younger (63.2 vs 65.3 years; $P=.026)$ and had a higher proportion of women $(72.3 \%$ vs $40.9 \% ; P=.001)$ compared with primary patients without MRT. Conversely, primary matched patients without MRT had more prior strokes $(5.5 \%$ vs $0.9 \% ; P=.003)$ and endocarditis (5.4 vs $1.9 ; P=.029$ ). Reoperative matched patients with MRT had more peripheral vascular disease than reoperative patients without MRT (28.3\% vs $14.6 \%$; $P=.031)$.
The operative characteristics of both matched cohorts were not different with respect to the type of procedure ( $P=.73$ for primary and $P=.38$ for reoperative patients) (Table 5). Compared with reoperative patients without MRT, reoperative patients with MRT underwent longer perfusion (248 vs 198 minutes; $P=.002$ ) and crossclamp times (146 vs 114 minutes; $P=.037$ ).

\section{Postoperative Outcomes of the Matched Primary and Reoperative Patients}

Postoperative outcomes for primary and reoperative valve surgery in matched patients with and without MRT are shown in Table 6. Primary patients with a history of MRT had a higher operative mortality compared with nonradiated patients $(3.8 \%$ vs $0.8 \% ; P=.004)$. For nonradiated primary patients, the observed-to-expected STS mortality ratio was 0.32 compared with 1.48 in patients with previous MRT. Also, primary patients with previous MRT had a higher incidence of deep sternal wound infections $(1.9 \%$ vs $0.6 \% ; P=.89)$, new onset renal insufficiency $(6.1 \%$ vs $2.2 \% ; P=.005)$, postoperative red blood cell transfusions (32.4\% vs $24.5 \% ; P=.023)$, and longer length of stay in an intensive care unit (51 days vs 47 days; $P=.003$ ), compared with their nonradiated primary counterparts.

A higher operative mortality was also present in reoperative MRT patients compared with their nonradiated 
TABLE 4. Postoperative data and in-hospital outcomes

\begin{tabular}{|c|c|c|c|c|}
\hline Outcome & All patients $(n=261)$ & Reoperative $(n=47)$ & Primary $(n=214)$ & $P$ value \\
\hline \multicolumn{5}{|l|}{ Postoperative complications } \\
\hline Return to OR & $8(3.1)$ & $4(8.5)$ & $4(1.9)$ & .037 \\
\hline Reoperation for bleeding & $7(2.7)$ & $3(6.4)$ & $4(1.9)$ & .118 \\
\hline Reoperation of valve & $1(0.4)$ & $1(2.1)$ & $0(0.0)$ & .180 \\
\hline Permanent stroke & $13(5.0)$ & $4(8.5)$ & $9(4.2)$ & .260 \\
\hline New onset renal insufficiency & $23(8.8)$ & $10(21.3)$ & $13(6.1)$ & .003 \\
\hline \multicolumn{5}{|l|}{ In-hospital outcomes } \\
\hline $\mathrm{PO} \mathrm{pRBC}$ & $98(37.5)$ & $29(61.7)$ & $69(32.2)$ & .001 \\
\hline PO FFP & $43(16.5)$ & $16(34.0)$ & $27(12.6)$ & .001 \\
\hline PO platelets & $24(9.2)$ & $10(21.3)$ & $14(6.5)$ & .004 \\
\hline Total mechanical ventilation time (h) & $7.5(4-15)$ & $20.3(6-79)$ & $7.0(4-12)$ & .001 \\
\hline Mechanical ventilation $\leq 24 \mathrm{~h}$ & $43(16.5)$ & $22(46.8)$ & $21(9.8)$ & .001 \\
\hline ICU stay (h) & $63(28-131)$ & $122(61-328)$ & $51(28-107)$ & .001 \\
\hline Postoperative LOS (d) & $8(6-16)$ & $15(9-27)$ & $7(5-14)$ & .001 \\
\hline Operative mortality & $16(6.1)$ & $8(17.0)$ & $8(3.7)$ & .003 \\
\hline
\end{tabular}

Values are presented as $\mathrm{n}(\%)$ or median (IQR). OR, Operating room; $P O$, postoperative; $p R B C$, pack of red blood cells; $F F P$, fresh frozen plasma; ICU, intensive care unit; $L O S$, length of stay.

matches $(17.4 \%$ vs $2.3 \% ; P=.001)$, which resulted in observed-to-expected STS mortality ratios of 3.35 and 0.45 , respectively. Overall, reoperative MRT patients had higher incidence of postoperative adverse events such as stroke $(8.7 \%$ vs $2.8 \% ; P=.081)$ and new-onset renal insufficiency $(19.6 \%$ vs $8.9 \% ; P=.062)$. Reoperative MRT patients were transfused with more fresh frozen plasma $(32.6 \%$ vs $17.8 \% ; P=.042)$ and platelets

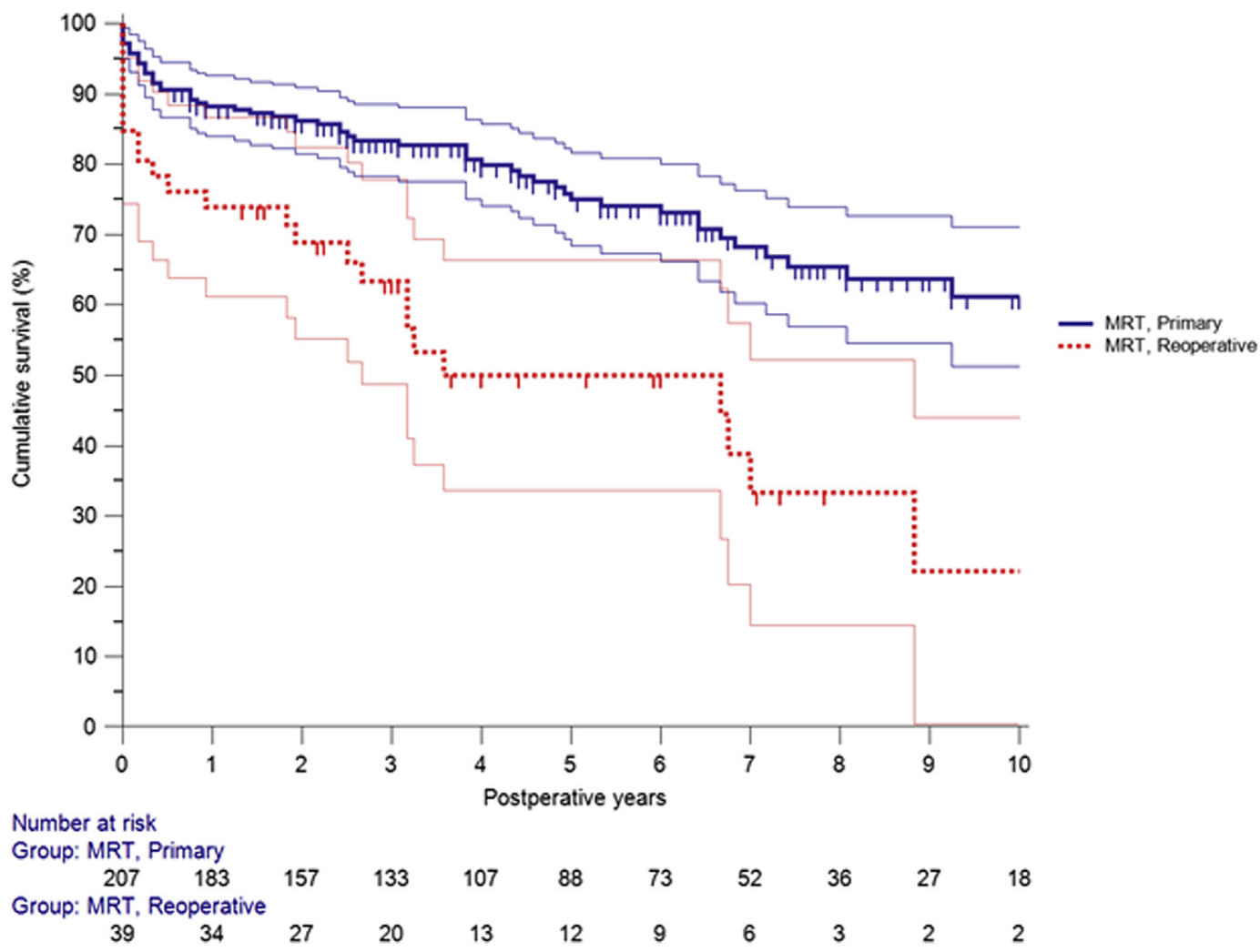

FIGURE 1. Kaplan-Meier survival curves for primary and reoperative patients with mediastinal radiation. Reoperative patients with mediastinal radiationinduced valvular heart disease had significantly shorter survival compared with patients without a previous sternotomy. MRT, Mediastinal radiotherapy. 
TABLE 5. Baseline and operative characteristics of matched primary and reoperative patients with and without radiation-induced valvular heart disease

\begin{tabular}{|c|c|c|c|c|c|c|}
\hline \multirow[b]{2}{*}{ Demographic characteristic } & \multicolumn{2}{|c|}{ Matched primary } & \multirow[b]{2}{*}{$P$ value } & \multicolumn{2}{|c|}{ Matched reoperations } & \multirow[b]{2}{*}{$P$ value } \\
\hline & No MRT $(n=836)$ & $\overline{\text { MRT }(n=213)}$ & & No MRT (n = 213) & $\overline{\text { MRT }(n=46)}$ & \\
\hline Age (y) & $65.3 \pm 13.1$ & $63.2 \pm 12.1$ & .026 & $63.5 \pm 13.6$ & $60.1 \pm 13.0$ & .265 \\
\hline Women & $342(40.9)$ & $154(72.3)$ & .001 & $83(39.0)$ & $19(41.3)$ & .868 \\
\hline Diabetes & $153(18.3)$ & $34(16.0)$ & .483 & $55(25.8)$ & $13(28.3)$ & .715 \\
\hline Renal insufficiency & $33(3.9)$ & $9(4.2)$ & .845 & $23(10.8)$ & $5(10.9)$ & .999 \\
\hline Preoperative creatinine & $1.09 \pm 0.67$ & $0.98 \pm 0.42$ & .028 & $1.20 \pm 0.67$ & $1.17 \pm 0.41$ & .786 \\
\hline Peripheral vascular disease & $74(8.9)$ & $20(9.4)$ & .789 & $31(14.6)$ & $13(28.3)$ & .031 \\
\hline Cerebrovascular disease & $88(10.5)$ & $11(5.2)$ & .017 & $39(18.3)$ & $7(15.2)$ & .832 \\
\hline Cerebrovascular accident & $46(5.5)$ & $2(0.9)$ & .003 & $17(8.0)$ & $3(6.5)$ & 1.000 \\
\hline Endocarditis & $45(5.4)$ & $4(1.9)$ & .029 & $36(16.9)$ & $5(10.9)$ & .379 \\
\hline Congestive heart failure & $351(42.0)$ & $100(46.9)$ & .215 & $135(63.4)$ & $30(65.2)$ & .867 \\
\hline NYHA class III or IV & $304(36.4)$ & $76(35.7)$ & .873 & $112(52.6)$ & $27(58.7)$ & .516 \\
\hline Ejection fraction $(\%)$ & $60.0(55-65)$ & $60.0(50-65)$ & .269 & $55.0(50-65)$ & $55.0(45-60)$ & .084 \\
\hline STS PROM & $2.51 \pm 2.51$ & $2.57 \pm 2.15$ & .337 & $5.16 \pm 5.04$ & $5.20 \pm 5.36$ & .963 \\
\hline \multicolumn{7}{|l|}{ Operative data } \\
\hline Valve procedures & & & .737 & & & .383 \\
\hline Isolated AV & $286(34.2)$ & $67(31.4)$ & & $66(31.0)$ & $13(28.3)$ & \\
\hline $\mathrm{AV}+\mathrm{CABG}$ & $146(17.5)$ & $46(21.6)$ & & $19(8.9)$ & $6(12.8)$ & \\
\hline $\mathrm{AV}+$ other valve & $80(9.6)$ & $18(8.5)$ & & $15(15)$ & $3(6.4)$ & \\
\hline Isolated MV & $125(15.0)$ & $32(15.0)$ & & $20(9.4)$ & $7(14.9)$ & \\
\hline Other isolated valve & $4(0.5)$ & $1(0.5)$ & & $15(7.0)$ & $4(8.1)$ & \\
\hline Other valve + CABG & $52(6.2)$ & $11(5.2)$ & & $24(11.3)$ & $4(8.5)$ & \\
\hline Miscellaneous complex valve & $142(17.0)$ & $38(17.7)$ & & $54(25.3)$ & $10(21.3)$ & \\
\hline Perfusion time (min) & $125(93-172)$ & $131(98-180)$ & .239 & $198(144-293)$ & $248(191-390)$ & .002 \\
\hline Crossclamp time (min) & $92(67-133)$ & $92(67-133)$ & .746 & $114(81-170)$ & $146(94-214)$ & .037 \\
\hline \multicolumn{7}{|l|}{ Patients transfused in OR } \\
\hline $\mathrm{pRBC}$ & $148(17.7)$ & $69(32.4)$ & .001 & $94(44.1)$ & $27(58.7)$ & .076 \\
\hline FFP & $84(10.0)$ & $18(8.5)$ & .520 & $84(39.4)$ & $24(52.2)$ & .138 \\
\hline Platelets & $59(7.1)$ & $17(8.0)$ & .657 & $66(31.0)$ & $22(47.8)$ & .023 \\
\hline
\end{tabular}

Values are presented as $\mathrm{n}(\%)$, mean \pm standard deviation, or median (interquartile range). MRT, Mediastinal radiotherapy; NYHA, New York Heart Association; STS, Society of Thoracic Surgeons; $P R O M$, predicted risk of mortality; $A V$, aortic valve; $C A B G$, coronary artery bypass graft; $M V$, mitral valve; $O R$, operating room; $p R B C$, pack of red blood cells; $F F P$, fresh frozen plasma.

$(21.7 \%$ vs $10.3 \% ; P=.46)$, had longer ventilation time (20.3 hours vs 10 hours; $P=.003$ ), intensive care unit length of stay ( 120 hours vs 66 hours; $P=.003$ ), and overall length of stay ( 15 days vs 7 days; $P=.001$ ), compared with nonradiated reoperative patients.

\section{Adjusted Operative Mortality Analysis of the Matched Primary and Reoperative Patients}

Adjusted odds for operative mortality were higher in reoperative patients with MRT (odds ratio [OR], 12.96; 95\% CI, 4.97-33.78; $P=.001$ ), followed by primary patients with MRT (OR, 4.87; 95\% CI, 1.72-13.78; $P=.003$ ), reoperative patients without MRT (OR, 1.1; 95\% CI, $0.380-3.18 ; P=.851$ ), compared with primary patients without a history of MRT. Additional predictors of operative mortality in our model were age (OR, 1.06; $95 \%$ CI, 1.02-1.10; $P=.002)$ and renal insufficiency (OR, 4.92; 95\% CI, 1.84-13.15; $P=.001$ ) (Table 7).

\section{Long-term Survival of the Matched Primary and Reoperative Patients}

For all patients included in the primary and reoperative matched cohorts, there were 259 deaths during the study period. Mean survival was significantly lower for primary patients with MRT $(9.51 ; 95 \%$ CI, 8.68-10.34) compared with their matched counterparts without MRT (11 years; 95\% CI, 10.65-11.35; $P<.001)$. Similarly, reoperative patients with MRT had a significantly lower survival (5.7 years; 95\% CI, 4.01-7.55) compared with matched reoperative patients without prior MRT (9.46 years; $95 \%$ 
TABLE 6. Postoperative data and in-hospital outcomes of matched primary and reoperative patients with and without radiation-induced valvular heart disease

\begin{tabular}{|c|c|c|c|c|c|c|}
\hline \multirow[b]{2}{*}{ Outcome } & \multicolumn{2}{|c|}{$\begin{array}{l}\text { Matched primary } \\
\end{array}$} & \multirow[b]{2}{*}{$P$ value } & \multicolumn{2}{|c|}{ Matched reoperations } & \multirow[b]{2}{*}{$P$ value } \\
\hline & No MRT $(n=836)$ & MRT $(n=213)$ & & No MRT $(n=213)$ & MRT $(n=46)$ & \\
\hline \multicolumn{7}{|l|}{ Postoperative complications } \\
\hline Return to OR & $16(1.9)$ & $4(1.9)$ & .037 & $18(8.5)$ & $4(8.5)$ & .037 \\
\hline DSWI & $5(0.6)$ & $4(1.9)$ & .089 & $3(1.4)$ & $1(2.2)$ & .545 \\
\hline Sepsis & $11(1.3)$ & $4(1.9)$ & .521 & $4(1.9)$ & $1(2.2)$ & .999 \\
\hline Permanent stroke & $22(2.6)$ & $4(1.9)$ & .630 & $6(2.8)$ & $4(8.7)$ & .081 \\
\hline New onset renal insufficiency & $18(2.2)$ & $134(6.1)$ & .005 & $19(8.9)$ & 9 (19.6) & .062 \\
\hline \multicolumn{7}{|l|}{ In-hospital outcomes } \\
\hline \multicolumn{7}{|c|}{ Patients transfused postoperatively } \\
\hline $\mathrm{pRBC}$ & $205(24.5)$ & $69(32.4)$ & .023 & $107(50.2)$ & $28(60.9)$ & .198 \\
\hline FFP & $110(13.2)$ & $27(12.7)$ & .910 & $38(17.8)$ & $15(32.6)$ & .042 \\
\hline Platelets & $51(6.1)$ & $14(6.6)$ & .720 & $22(10.3)$ & $10(21.7)$ & .046 \\
\hline Total ventilation time & $6.5(4,11)$ & $6.9(4,12)$ & .001 & $10.0(5,17)$ & $20.3(6,86)$ & .003 \\
\hline Vent $\leq 24 \mathrm{~h}$ & $68(8.1)$ & $21(9.9)$ & .611 & $41(19.2)$ & $21(45.7)$ & .001 \\
\hline ICU stay (h) & $47(24,75)$ & $51(28,107)$ & .003 & $66(38,133)$ & $120(57,332)$ & .003 \\
\hline Postoperative LOS (d) & $7(5-10)$ & $7(5-14)$ & .003 & $9(6-14)$ & $15(9-27)$ & .001 \\
\hline Operative mortality & $7(0.8)$ & $8(3.8)$ & .004 & $5(2.3)$ & $8(17.4)$ & .001 \\
\hline O/E based on STS PROM & 0.32 & 1.48 & & 0.45 & 3.35 & \\
\hline
\end{tabular}

Values are presented as n (\%) or median (interquartile range). $M R T$, Mediastinal radiotherapy; $O R$, operating room; $D S W I$, deep sternal wound infection; $p R B C$, pack of red blood cells; FFP, fresh frozen plasma; ICU, intensive care unit; $L O S$, length of stay; O/E, observed to expected; STS, Society of Thoracic Surgeons; PROM, predicted risk of mortality.

CI, 8.59-10.33; $P<.001)$. Primary patients with MRT had a similar survival compared with reoperative patients with no history of MRT $(P=.89)$ (Figure 2).

\section{Adjusted Survival of the Matched Primary and Reoperative Patients}

A conditional Cox proportional hazards model was done to elicit risk factors associated with decreased survival in the matched cohorts (Figure 3 and Table 8). Compared with nonradiated primary patients, primary patients with prior MRT (hazard ratio [HR], 2.24; 95\% CI, 1.73-1.91; $P<.001)$ and reoperative patients with prior MRT (HR, $3.19 ; 95 \%$ CI, $1.95-5.21 ; P<.001)$ had a significantly higher risk of decreased survival. Additional predictors of

TABLE 7. Logistic regression analysis for operative mortality

\begin{tabular}{lrrrrr}
\hline & & & \multicolumn{2}{c}{$\mathbf{9 5 \%}$ Confidence } \\
& Odds & $\boldsymbol{P}$ & \multicolumn{2}{c}{ interval } \\
\cline { 4 - 6 } \multicolumn{1}{c}{ Predictor variable } & ratio & value & Low & High \\
\hline Group (vs primary patients & & .001 & & \\
$\quad$ without MRT) & & & & \\
Reoperative patients without MRT & 1.10 & .861 & 0.380 & 3.180 \\
Primary patients with MRT & 4.87 & .003 & 1.724 & 13.789 \\
$\quad$ Reoperative patients with MRT & 12.96 & .001 & 4.977 & 33.788 \\
Age & 1.06 & .002 & 1.021 & 1.103 \\
Renal insufficiency & 4.92 & .003 & 1.842 & 13.158 \\
\hline$M R T$, Mediastinal radiation therapy. & & & & \\
\end{tabular}

increased long-term mortality were age (HR, 1.04; 95\% CI, 1.03-1.05; $P<.001$ ), renal insufficiency (HR, 1.56; $95 \%$ CI, $1.15-2.11 ; P<.004)$, congestive heart failure (HR, 1.72; 95\% CI, 1.33-2.23; $P<.001$ ), peripheral vascular disease (HR, 1.33; 95\% CI, 1.02-272; $P=.030$ ), diabetes (HR, 1.61; 95\% CI 1.23-2.11; $P<.001$ ), and NYHA class III or IV (HR, 2.81; 95\% CI, 1.99-3.97; $P<.001)$. The type of cancer, gender, prior history of stroke, infectious endocarditis, and type of procedure were not contributory (all $P$ values $>$.17).

\section{DISCUSSION}

To our knowledge, this study represents the largest cohort of surgically treated patients with radiation-induced VHD. In this relatively young group of predominantly Hodgkin lymphoma and breast cancer survivors, aortic valve replacement was performed in more than three-quarters of all patients. Both primary and reoperative procedures for radiation-induced VHD resulted in worse outcomes than those predicted by the STS risk algorithm. Furthermore, in patients with prior MRT, reoperative surgery was associated with a $13 \%$ increase in operative mortality compared with primary surgery. These findings were emphasized after matching primary and reoperative patients with radiation-induced VHD to nonradiated counterparts. In matched patients who underwent primary valve procedures, MRT was associated with a higher operative 


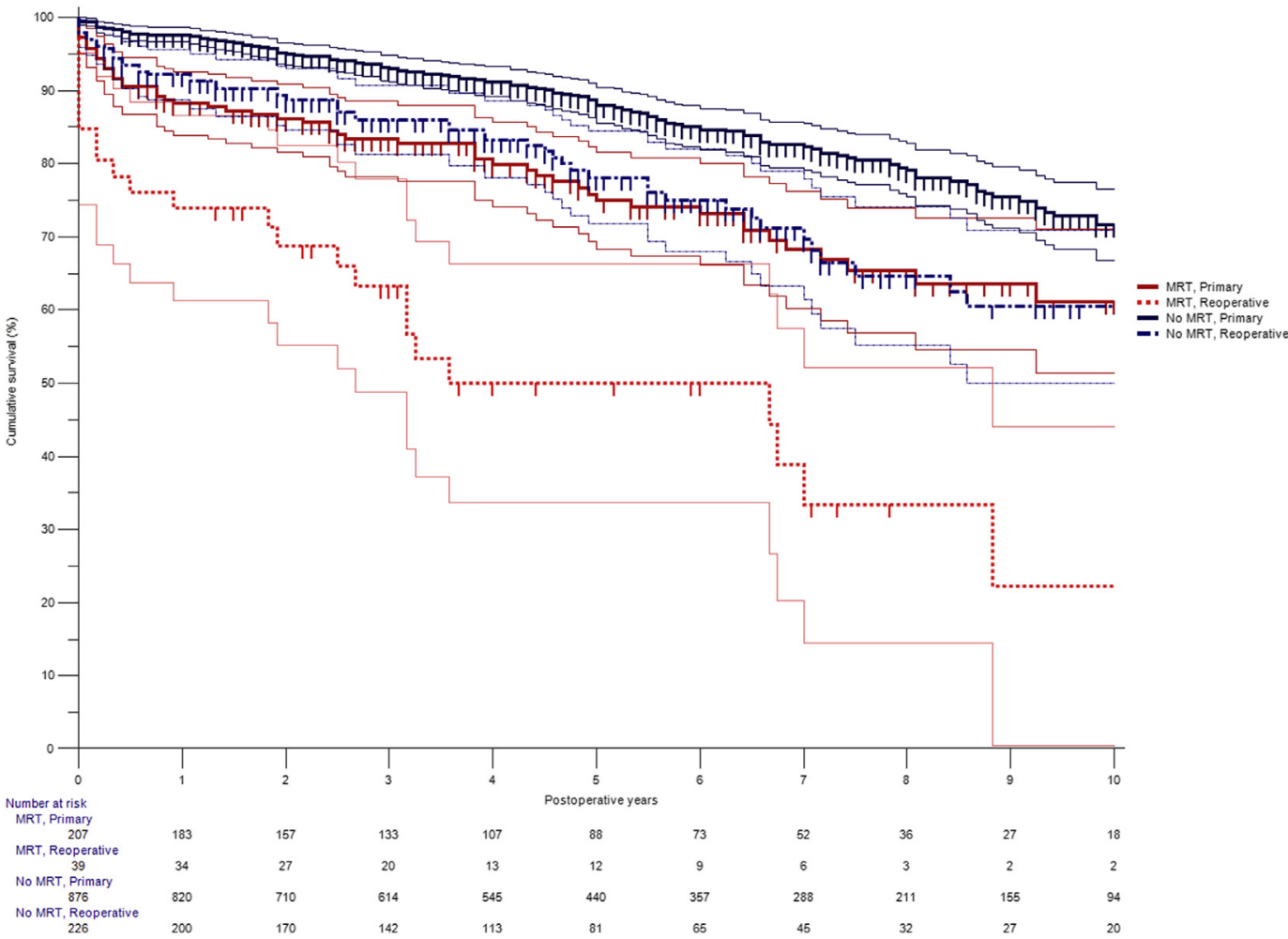

FIGURE 2. Kaplan-Meier survival curves for matched primary and reoperative patients with and without prior mediastinal radiation. The lowest cumulative survival was observed in reoperative, followed by primary patients with mediastinal radiotherapy (MRT), compared with their matched counterparts without MRT. Primary patients with MRT had a similar survival compared with reoperative patients with no history of MRT $(P=.89)$.

mortality $(3.8 \%$ vs $0.8 \%)$ and a 2.2 -fold higher risk of decreased long-term survival (HR, 2.24). Similarly, in the reoperative matched cohort, MRT patients had substantially worse outcomes with respect to operative mortality $(2.3 \%$ vs $17.4 \%$ ) and decreased long-term survival (HR, 3.19) compared with primary nonradiated patients. Our results underscore the unfavorable outcomes associated with surgery for radiation-induced VHD and emphasize the underestimation of operative risk in these patients when relying solely on the STS PROM, especially for patients undergoing reoperation. These findings should persuade physicians to carefully evaluate the risks associated with surgical treatment in these patients, with consideration toward less-invasive therapeutic modalities, including transcatheter-based approaches.

\section{Outcomes of Radiation-Induced VHD}

Radiation-induced cardiac disease will become progressively more relevant as long-term cancer survival continues to improve ${ }^{17}$ The time interval from radiation to surgery ranges from 10 to 25 years and these patients frequently present at a younger age relative to the general population, especially for radiation-associated aortic valve replacements. ${ }^{2,11,18,19}$ Indeed, the average age of our radiated cohort was 62.6 years compared with 76 years in the STS
Adult Cardiac Surgery Database..$^{20}$ Our observed pattern for radiation-induced VHD was consistent with the literature, ${ }^{11}$ with a high prevalence of aortic valve disease $(77 \%)$, with mixed stenotic and regurgitant lesions, followed by mitral valve disorders. In our study, less than half of surgeries were isolated valve replacements ( $30 \%$ aortic valve replacement and $15 \%$ mitral valve replacement), and the majority were a combination of concomitant valve procedures or complex valve surgeries, exemplifying the ubiquitous nature of radiation-induced heart disease.

Total radiation dose is a determinant in the development of radiation-induced VHD. ${ }^{21}$ Chang and colleagues ${ }^{7}$ used type of cancer as a surrogate for radiation field and dose, and showed improved outcomes in patients exposed to tangential compared with extensive radiation. Although the exact radiation dose and the time to development of valvular disease could not be determined in our study, operative mortality was not significantly different when comparing breast cancer to Hodgkin/thymic/testicular cancers, and type of cancer was not found to be contributory on unadjusted survival analyses. Furthermore, multivariate modeling failed to show a significant change in mortality risk based on cancer type. However, the high frequency of subjects who underwent mediastinal radiation for Hodgkin lymphoma $(48 \%)$ and breast cancer $(36 \%)$, 


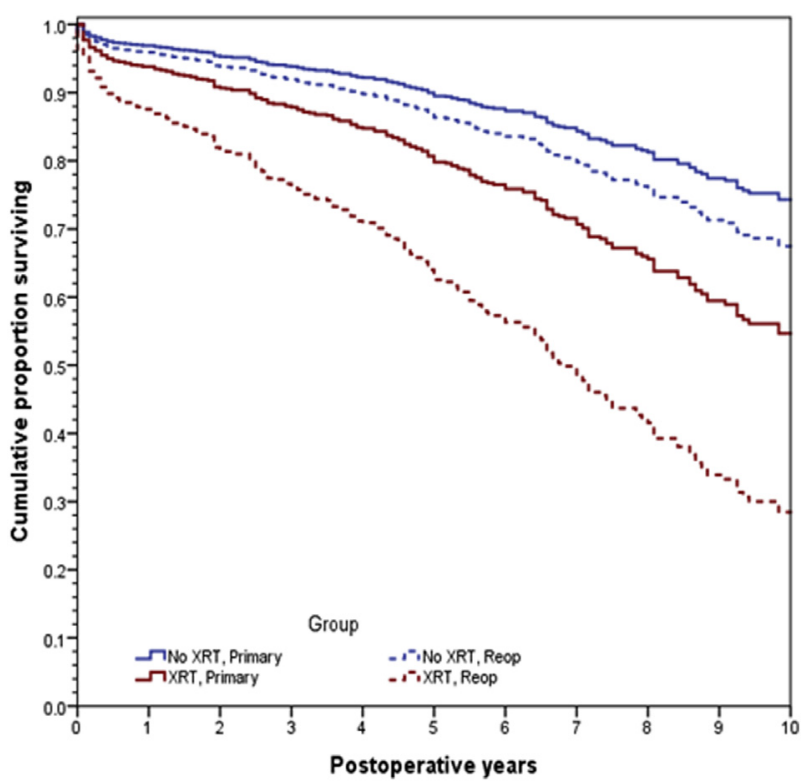

FIGURE 3. Conditional Cox proportional hazards modeling survival curves. Primary patients with prior mediastinal radiotherapy (MRT) (hazard ratio, 2.24; 95\% confidence interval, 1.73-1.91; $P<.001$ ) and reoperative patients with prior MRT (hazard ratio, 3.19; $95 \%$ confidence interval, 1.95-5.21; $P<.001$ ) had a significantly lower adjusted survival, compared with nonradiated primary matches. XRT, Radiotherapy; Reop, reoperation.

emphasizes the contemporary relevance of radiationinduced VHD in this patient population, ${ }^{9,22}$ even with considerable advancements in limiting radiation field and total dose.

\section{Outcomes of Primary Cases}

Our overall $6 \%$ operative mortality compares favorably with previous reports of cardiac surgery in patients with MRT. ${ }^{3,5,6,23}$ Handa and colleagues ${ }^{5}$ described 60 cases of radiation-induced VHD where aortic valve replacement was also the most frequent procedure. A smaller series by Crestanello and colleagues ${ }^{3}$ focused on the surgical experience with atrioventricular valves-14 mitral and 6 tricuspid repairs-in which they observed a high rate of early repair failure. The latter series was composed mainly of complex procedures, with only 5 isolated valve repairs. Early surgical mortality for these studies was $12 \%$ to $14 \%$ for valve surgery. ${ }^{3,5}$ Chang and colleagues ${ }^{7}$ published the largest series to date, with 230 patients who underwent cardiac surgery from 2000 to 2005 and were exposed to different intensities of chest radiation. Their operative mortality ranged from $2.4 \%$ to $13 \%$ depending on the radiation field. A recent publication by $\mathrm{Wu}$ and colleagues ${ }^{16}$ included 173 surgical cases (coronary and valve, both isolated and concomitant) with radiation-associated heart disease that were matched to 305 patients without chest radiation. The radiated group experienced a significantly higher mortality $(4 \%)$ compared with the comparison group $(0.3 \%)$. Despite the heterogeneity of these studies, there is consistency in the increased mortality risk conveyed by MRT.

In our study, compared with nonradiated matches, patients who underwent primary valve surgery for radiation-induced VHD had a substantially higher operative mortality $(3.8 \%$ vs $0.8 \%$ ) despite a similar STS PROM (2.51 and 2.57, respectively), resulting in a higher observed-to-expected STS mortality ratio (1.48 vs 0.32 ). These findings we further supported by the observed 4.8 -fold increase in the odds of operative mortality in patients with prior MRT compared with nonradiated matches.

The estimated 10-year survival of our cohort was 55\%. Previously reported 5-year survival is $66 \%{ }^{5}$ and others have published similar long-term results with varying follow-ups. ${ }^{7,16}$ We observed a shorter cumulative survival in patients with MRT who underwent primary valve procedures compared to their nonradiated matches. Similar findings have been reported in other studies, in which patients with radiation-induced heart disease have

TABLE 8. Conditional Cox proportional hazard model for long-term survival

\begin{tabular}{|c|c|c|c|c|}
\hline \multirow[b]{2}{*}{ Predictor variable } & \multirow[b]{2}{*}{ Hazard ratio } & \multirow[b]{2}{*}{$P$ value } & \multicolumn{2}{|c|}{ 95\% Confidence interval } \\
\hline & & & Low & High \\
\hline Group (vs primary patients without MRT) & & .001 & & \\
\hline Reoperative patients without MRT & 1.16 & .418 & 0.806 & 1.679 \\
\hline Primary patients with MRT & 2.24 & .001 & 1.738 & 2.911 \\
\hline Reoperative patients with MRT & 3.19 & .001 & 1.959 & 5.215 \\
\hline Age & 1.04 & .001 & 1.030 & 1.054 \\
\hline Diabetes & 1.61 & .001 & 1.236 & 2.117 \\
\hline Congestive heart failure & 1.72 & .001 & 1.330 & 2.232 \\
\hline NYHA class III or IV & 2.81 & .001 & 1.998 & 3.971 \\
\hline Renal insufficiency & 1.56 & .004 & 1.158 & 2.115 \\
\hline Peripheral vascular disease & 1.33 & .030 & 1.028 & 1.725 \\
\hline
\end{tabular}

MRT, Mediastinal radiation therapy; NYHA, New York Heart Association. 
been consistently marked by decreased survival, relative to both matched population life tables ${ }^{7}$ and matched controls. ${ }^{16}$

\section{Outcomes of Reoperative Cases}

Reoperative valve surgeries are more technically demanding than primary procedures and are associated with worse outcomes, even in nonradiated patients. ${ }^{24-26}$ Reoperative valve surgery in a radiated chest poses increased complexity from both technical and medical perspectives. Technical difficulties include denser adhesions, smaller valve annulus size, and fibrotic tissues, which result in longer cardiopulmonary bypass and aortic crossclamp times. Further inflammatory insults, due to extensive dissection during the procedure, result in a higher incidence of postoperative pleural effusions and third spacing of fluids, necessitating longer hospital stays.

The reoperative patients with prior MRT were more complex, with a higher burden of comorbidities and a higher mean STS PROM (5.21) compared with primary patients with MRT (2.58). Despite this difference, their STS PROM considerably underestimated the observed mortality by 3.2 -fold. ${ }^{16}$ Although reoperative-matched patients with and without prior MRT had similar STS PROM (5.20 and 5.16, respectively), operative mortality was substantially higher in the former group $(17.4 \%$ and $2.3 \%$ ). This resulted in a high observed to expected STS mortality ratio in reoperative patients with prior MRT, but not in their nonradiated counterparts (3.35 and 0.25, respectively). It is beyond the scope and reach of this article to assess the performance, both calibration and discrimination, of the STS PROM in these patients, but this type of analysis might be possible with the addition of mediastinal radiation to the updated version 2.81 STS data collection form. However, our findings emphasize the underperformance of the STS PROM in patients with radiation-induced VDH, particularly in reoperative cases.

In our study, the shortest cumulative survival was present in patients with a history of mediastinal radiation and a prior sternotomy (5.78 years), which was significantly shorter compared with both nonradiated reoperative matches (9.46 years) and primary patients with prior MRT (9.51 years). Wu and colleagues ${ }^{16}$ also described an important difference in longitudinal survival (with a mean follow-up of 7.6 years) between reoperative $(71 \%)$ and primary $(51 \%)$ patients with prior MRT.

\section{Factors Influencing Long-term Survival}

Primary and reoperative patients with radiation-induced VHD had more than 2 and 3 times the risk of long-term death, respectively, compared with nonradiated matched patients in our conditional adjusted survival model. The increased risk of long-term mortality conferred by reoperation was not present in nonradiated matched patients. Our results show that mediastinal radiation is a significant risk factor for worse long-term survival and that a concomitant prior sternotomy confers a higher risk that is not observed in nonradiated matched patients. Additional predictors of long-term survival were age, renal insufficiency, congestive heart failure, diabetes, peripheral vascular disease, and NYHA class III or IV. Similar to our findings, previously described factors associated with decreased long-term survival in MRT patients are constrictive pericarditis, left ventricular ejection fraction $<50 \%$, NYHA class IV symptoms of heart failure, and prolonged cardiopulmonary bypass time. ${ }^{3,5} \mathrm{Wu}$ and colleagues ${ }^{16}$ reported previous cardiac surgery and diabetes to be significantly associated with increased mortality. However, previous cardiac surgery was not a significant predictor of increased mortality in their adjusted survival analysis. The updated American College of Cardiology/ American Heart Association guidelines include radiation as a procedure-specific impediment for cardiac surgery, ${ }^{27}$ but with the poor surgical outcomes among reoperative cases we suggest that reoperation in the context of MRT may be a prohibitive risk factor for surgery.

\section{Alternative Treatment Options}

Heart transplantation has been previously advocated for patients with prior MRT, especially for those with concomitant radiation-induced pulmonary disease. However, there is continuing concern about the administration of immunosuppressive agents in patients with a positive cancer history. Although the low number of deaths due to cancer ${ }^{11}$ and low prevalence or recurrence at the time of surgery ${ }^{17}$ in these patients might appear encouraging, extrapolating data from patients without immunomodulation therapy to posttransplant patients has serious caveats. Nevertheless, early reports on a small number of heart transplants in patients with prior chest radiation have yielded promising results. ${ }^{23}$

Conservative treatment in high-risk surgical patients is a reasonable option and an argument could be made for delaying surgical intervention in reoperative patients with prior mediastinal radiation. However, one can argue that waiting allows for the development of a more significant congestive heart failure, deriving from their underlying cardiomyopathy, which would result in even worse outcomes.

Emerging alternatives such as transcatheter-based therapies may offer viable alternatives to surgery in these patients. Transcatheter aortic valve replacement is a valid option for high-risk patients, and with the high prevalence of aortic stenosis in MRT patients we believe that reoperative patients with radiation-induced VHD may derive particular benefit from this new intervention. In a small series, Bouleti and colleagues ${ }^{28}$ showed similar short-term mortality ( $8 \%$ for both) and 5-year survival (33\% vs $42 \% ; P=.26$ ) between 26 transcatheter aortic 
valve replacement patients with radiation-induced VHD and 26 nonradiated matched transcatheter aortic valve replacement controls. Despite the lack of large patient series and long-term follow-up data, it is obvious that transcatheter therapies should be included in the armamentarium of valve treatments offered to patients with MRT. Burgeoning technologies such as transcatheter mitral valve replacements and repairs will likely be used for other radiation-induced valvular pathologies in the future.

\section{Limitations}

The current study has limitations related to its retrospective, observational design and as a single center study there are inherent disadvantages, especially related to selection bias, that may influence the generalizability of our findings to other populations. We were unable to ascertain the patients' cancer stage, radiation field, and radiation dose or the time interval to the index surgery, all of which might have influenced surgical outcomes. Although the type of cancer was not a contributory factor in unadjusted and multivariable survival analyses, our findings may not apply to other populations of patients with MRT. Because the cause of death data were unavailable for longitudinal survival analyses, we cannot evaluate how cancer recurrence may have contributed to long-term outcomes. Although reoperative cardiac surgery was identified as a significant risk factor associated with reduced postoperative survival, the current study design cannot determine whether this relationship is directly causal, or describe precise mechanisms resulting in our findings. Lastly, due to the inconsistent reporting of preoperative echocardiography findings, especially at the beginning of our observation period, an analysis of preoperative cardiac output and its association with long-term outcomes in patients with radiation-induced VHD was not possible and therefore remains as a potential confounder. Our results should be interpreted with these cautions.

\section{CONCLUSIONS}

To our knowledge, this cohort represents the largest experience to date in the surgical management of radiation-induced VHD. Preoperative assessment based only on STS PROM might underestimate these patients' true surgical risk, both with primary and reoperative surgeries. In particular, reoperations are associated with significantly higher operative and long-term mortality. Our results emphasize the need for careful preoperative risk assessment. Further studies are needed to assess the potential benefit of using nonsurgical approaches such as transcatheter aortic valve replacement in these patients.

\section{Conflict of Interest Statement}

Authors have nothing to disclose with regard to commercial support.

\section{References}

1. Benoff LJ, Schweitzer P. Radiation therapy-induced cardiac injury. Am Heart J. 1995;129:1193-6.

2. Carlson RG, Mayfield WR, Normann S, Alexander JA. Radiation-associated valvular disease. Chest. 1991;99:538-45.

3. Crestanello JA, McGregor CG, Danielson GK, Daly RC, Dearani JA, Orszulak TA, et al. Mitral and tricuspid valve repair in patients with previous mediastinal radiation therapy. Ann Thorac Surg. 2004;78:826-31.

4. Hancock SL, Donaldson SS, Hoppe RT. Cardiac disease following treatment of Hodgkin's disease in children and adolescents. J Clin Oncol. 1993;11:1208-15.

5. Handa N, McGregor CG, Danielson GK, Daly RC, Dearani JA, Mullany CJ, et al. Valvular heart operation in patients with previous mediastinal radiation therapy. Ann Thorac Surg. 2001;71:1880-4.

6. Handa N, McGregor CGA, Danielson GK, Orszulak TA, Mullany CJ, Daly RC, et al. Coronary artery bypass grafting in patients with previous mediastinal radiation therapy. J Thorac Cardiovasc Surg. 1999;117:1136-43.

7. Chang AS, Smedira NG, Chang CL, Benavides MM, Myhre U, Feng J, et al. Cardiac surgery after mediastinal radiation: extent of exposure influences outcome. $J$ Thorac Cardiovasc Surg. 2007;133:404-13.

8. Hull MC, Morris CG, Pepine CJ, Mendenhall NP. Valvular dysfunction and carotid, subclavian, and coronary artery disease in survivors of hodgkin lymphoma treated with radiation therapy. JAMA. 2003;290:2831-7.

9. Galper SL, Yu JB, Mauch PM, Strasser JF, Silver B, Lacasce A, et al. Clinically significant cardiac disease in patients with Hodgkin lymphoma treated with mediastinal irradiation. Blood. 2011;117:412-8.

10. Dijos M, Reynaud A, Leroux L, Reant P, Cornolle C, Roudaut R, et al. Efficacy and follow-up of transcatheter aortic valve implantation in patients with radiation-induced aortic stenosis. Open Heart. 2015;2:e000252.

11. Heidenreich PA, Hancock SL, Lee BK, Mariscal CS, Schnittger I. Asymptomatic cardiac disease following mediastinal irradiation. J Am Coll Cardiol. 2003;42: 743-9.

12. Jaworski C, Mariani JA, Wheeler G, Kaye DM. Cardiac complications of thoracic irradiation. J Am Coll Cardiol. 2013;61:2319-28.

13. Siregar S, de Heer F, van Herwerden LA. Cardiac surgery in patients irradiated for Hodgkin's lymphoma. Neth Heart J. 2010;18:61-5.

14. Shahian DM, O'Brien SM, Filardo G, Ferraris VA, Haan CK, Rich JB, et al. The Society of Thoracic Surgeons 2008 cardiac surgery risk models: part 3-valve plus coronary artery bypass grafting surgery. Ann Thorac Surg. 2009;88(1 Suppl):S43-62.

15. Shahian DM, O'Brien SM, Filardo G, Ferraris VA, Haan CK, Rich JB, et al. The Society of Thoracic Surgeons 2008 cardiac surgery risk models: part 1-coronary artery bypass grafting surgery. Ann Thorac Surg. 2009; 88(1 Suppl):S2-22.

16. Wu W, Masri A, Popovic ZB, Smedira NG, Lytle BW, Marwick TH, et al. Longterm survival of patients with radiation heart disease undergoing cardiac surgery: a cohort study. Circulation. 2013;127:1476-85.

17. Veinot JP, Edwards WD. Pathology of radiation-induced heart disease: a surgical and autopsy study of 27 cases. Hum Pathol. 1996;27:766-73.

18. Adams MJ, Lipshultz SE, Schwartz C, Fajardo LF, Coen V, Constine LS Radiation-associated cardiovascular disease: manifestations and management Semin Radiat Oncol. 2003;13:346-56.

19. Veeragandham RS, Goldin MD. Surgical management of radiation-induced heart disease. Ann Thorac Surg. 1998;65:1014-9.

20. Brennan JM, Edwards FH, Zhao Y, O'Brien SM, Douglas PS, Peterson ED Long-term survival after aortic valve replacement among high-risk elderly patients in the United States: insights from the Society of Thoracic Surgeons Adult Cardiac Surgery Database, 1991 to 2007. Circulation. 2012;126:1621-9.

21. Senkus-Konefka E, Jassem J. Cardiovascular effects of breast cancer radiotherapy. Cancer Treat Rev. 2007;33:578-93.

22. McGale P, Darby SC, Hall P, Adolfsson J, Bengtsson N-O, Bennet AM, et al. Incidence of heart disease in 35,000 women treated with radiotherapy for breast cancer in Denmark and Sweden. Radiother Oncol. 2011;100:167-75.

23. Handa N, McGregor CG, Daly RC, Dearani JA, Edwards BS, Frantz RP, et al. Heart transplantation for radiation-associated end-stage heart failure. Transpl Intl. 2000;13:162-5.

24. Davierwala PM, Borger MA, David TE, Rao V, Maganti M, Yau TM. Reoperation is not an independent predictor of mortality during aortic valve surgery. J Thorac Cardiovasc Surg. 2006;131:329-35.

25. Odell JA, Mullany CJ, Schaff HV, Orszulak TA, Daly RC, Morris JJ. Aortic valve replacement after previous coronary artery bypass grafting. Ann Thorac Surg. 1996;62:1424-30. 
26. Sundt TM III, Murphy SF, Barzilai B, Schuessler RB, Mendeloff EN, Huddleston CB, et al. Previous coronary artery bypass grafting is not a risk factor for aortic valve replacement. Ann Thorac Surg. 1997;64:651-8.

27. Nishimura RA, Otto CM, Bonow RO, Carabello BA, Erwin JP III, Guyton RA. 2014 AHA/ACC guideline for the management of patients with valvular heart disease: a report of the American College of Cardiology/American Heart Association Task Force on Practice Guidelines. J Thorac Cardiovasc Surg. 2014;148:e1-132.
28. Bouleti C, Amsallem M, Touati A, Himbert D, Iung B, Alos B, et al. Early and late outcomes after trans-catheter aortic valve implantation in patients with previous chest radiation. Heart. 2016;102:1044-51.

Key Words: mediastinal radiotherapy, valvular heart disease, radiation-induced valvular heart disease, reoperative valve surgery

Readers who found these articles interesting may also like to read the following papers found in recent and future issues of our sister publications, Seminars in Thoracic and Cardiovascular Surgery and Operative Techniques in Thoracic and Cardiovascular Surgery!

\section{Acquired: Aortic Valve}

STATE OF THE ART: Aortic Valve Replacement: Are We Spoiled for Choice? Mohamad Bashir. Semin Thoracic Surg 2017: In press. STATE OF THE ART: Sutureless Sorin Perceval Aortic Valve Implantation Steffen Pfeiffer. Semin Thoracic Surg 2017: 1-7.

ORIGINAL SUBMISSION: Aortic Valve Replacement Using a Perceval Sutureless Aortic Bioprosthesis David Heimansohn. Oper Tech Thorac Cardiovasc 2016: In Press.

ORIGINAL SUBMISSION: How to Perform a Simplified Technique for Transapical Aortic Valve Implantations Pierre-Yves Litzler. Oper Tech Thorac Cardiovasc 2016: 89-111.

ORIGINAL SUBMISSION: Comparison of Aortic Annulus Dimension after Aortic Valve neo-cuspidization with valve replacement and normal valve. Yoshitaka Yamamoto. Semin Thoracic Surg 2017: 143-149. 